\title{
COMMENTS ON THE CLUSTER MAIN-SEQUENCE FITTING METHOD. III. EMPIRICAL UBV REDDENING LINES FOR EARLY-TYPE STARS
}

\author{
David G. TURner \\ Department of Astronomy, Saint Mary's University, Halifax, Nova Scotia B3H 3C3, Canada \\ Received 10 July 1989; revised 15 August 1989
}

\begin{abstract}
Reddening line parameters in the $U B V$ system have been derived for early-type stars in six restricted $\left(2^{\circ}-3^{\circ}\right.$ radius ) Milky Way fields associated with open clusters and/or associations. The curvature terms are very similar from one field to another, with mean $\langle Y\rangle=0.021 \pm 0.006$, and indicate that there is very little (almost negligible) curvature in the reddening lines for OB stars. The slope terms differ quite significantly from one field to another over a range of at least $X=0.62$ to 0.80 , implying that mean galactic reddening relations should not be used for dereddening $U B V$ data for individual stars. No significant spectral type dependence of $X$ is found for O-, B-, and A-type stars, contrary to published results. A correlation of $\lambda_{\max }$ with $X$ for extinction by nearby dust clouds indicates that galactic variations in reddening line slope are likely to be due to variations in dust grain size distributions along different galactic lines of sight.
\end{abstract}

\section{INTRODUCTION}

A fundamental problem faced by users of Johnson and Morgan's (1953) broadband $U B V$ system is the correct determination of the effects of interstellar reddening on observed $U-B$ and $B-V$ colors. These effects are usually described by a relationship, known as the reddening line, which is of the form

$$
\mathrm{E}_{U-B} / \mathrm{E}_{B-V}=X+Y\left(\mathrm{E}_{B-V}\right) \text {. }
$$

In this equation, $\mathrm{E}_{U-B}$ and $\mathrm{E}_{B-V}$ are the color excesses in $U-B$ and $B-V, X$ is the slope of the reddening line, and $Y$ is the curvature term. $X$ and $Y$ are larger for late-type stars than for early-type stars due to a spectral type dependence of the effective wavelengths for the $U B V$ passbands. Their exact values have been established by two different methods, namely (i) from observations using stars of known intrinsic color which are affected by a wide variety of reddenings, and (ii) from calculations involving the convolution of stellar flux distributions with the interstellar reddening function and the wavelength response functions for the $U B V$ filtertelescope-photomultiplier combinations. Studies of this type have generally yielded values of $X \simeq 0.70$ for early-type stars, and corresponding values of $Y \simeq 0.05$. Somewhat different values have occasionally been derived by some researchers, but these results seem to have been overlooked by others.

The observational approach to examining $U B V$ reddening lines [method (i)] is described in two extensive investigations by Serkowski (1963) and FitzGerald (1970). Both studies produced tables of intrinsic stellar $U B V$ colors from the analysis of published observational material, and solved for $X$ and $Y$ by means of least-squares analyses involving large samples of stars of selected spectral type located in rather extensive areas of sky. FitzGerald's study, which considered stars of all spectral types and which was based upon a larger observational sample, yielded a mean value of $X=0.70 \pm 0.01$ for early-type stars, with indications of a spectral type dependence for B stars. A larger value of $X=0.75 \pm 0.01$ was obtained for the Cygnus region, which has always been considered to be anomalous. $Y$ was adopted from theoretical studies to be 0.05 , since least-squares solutions yielded contradictory values of $Y=0.06 \pm 0.01$ for $O-$
B0.5 stars and $-0.02 \pm 0.02$ for B1-B4 stars. FitzGerald has shown that this last result is probably spurious. Although Wampler $(1961,1962)$ and others have presented evidence for a galactic longitude dependence of $X$, this was not confirmed in the studies by Serkowski and FitzGerald. Likewise, Wampler's (1964) arguments that $Y \simeq 0.00$ were considered to be in conflict with theoretical predictions.

The theoretical treatment of reddening in the $U B V$ system [method (ii)] was initiated by Blanco $(1956,1957)$, but has been refined in several subsequent studies (see GutiérrezMoreno and Moreno 1975; Straizys et al. 1976; Crawford and Mandwewala 1976; Buser 1978) in order to take better account of atmospheric influences on the $U B V$ response functions. Wampler (1964) suggested several years ago that the theoretical approach tends to ignore the observational properties of the $U B V$ system, which depends upon standard stars to calibrate an instrumental system. These comments are still valid, particularly in light of the recent discussion by Popper (1982) outlining why photometrists occasionally find it difficult to match observations precisely to the Johnson system using instrumental systems that otherwise appear to satisfy the requirements for $U B V$ photometry (Johnson 1963). Systematic errors in $U B V$ photometry, obtained using instrumental systems that are not satisfactorily matched to Johnson's system, are frequently encountered in published data. For this reason, theoretical studies of interstellar reddening, which produce results similar but not always exactly matched to observational $U B V$ data, may not give truly representative values for the $X$ and $Y$ parameters.

The present investigation was undertaken in response to the realization that $U B V$ studies of open clusters by different researchers often result in rather disparate values of reddening and distance for the same cluster depending upon how the data were corrected for interstellar reddening. Such anomalies do not usually produce grossly different estimates for the luminosities of specific cluster members (e.g., Cepheids), since these are derived relative to main-sequence stars in the same clusters. However, there is always the concern that some of the currently practiced methods of treating interstellar reddening may produce results that are inherently erroneous (see discussions by Turner 1981a,b). This paper addresses the problem by examining the reddening in a 
few areas of the Milky Way where the exact form of Eq. (1) can be delineated.

\section{METHODOLOGY}

The approach adopted in this paper was to examine isolated regions of the Milky Way, generally of $2^{\circ}$ or $3^{\circ}$ radius, in which the reddening values for OB stars with well-determined photoelectric photometry and MK spectral types ranged over an interval of at least $1 \mathrm{~m} 0$ in $\mathrm{E}_{B-V}$. A baseline of this size was considered essential for proper statistical analysis of the reddening lines. The analysis was also restricted primarily to stars of spectral type B3 or earlier, although B supergiants of later type were included if their intrinsic colors were considered to be well established. An exception was made in the case of Upper Scorpius, which contains B and A dwarfs of all types that have been investigated in some detail by Garrison (1967).

The criteria used to select stars for the individual reddening samples were somewhat more restrictive than those adopted by Serkowski (1963) and FitzGerald (1970). In particular, published $U B V$ photometry was used only if it was closely tied to the Johnson system (e.g., observations by Hiltner, Johnson, and other experienced photometrists working with 1P21 photomultipliers). Most of the MK types adopted originated from researchers well versed in spectral classification (e.g., the Morgan-Keenan school), although other sources were used if good stellar photometry was also available. This point is discussed further in Sec. III. Certain types of stars, namely emission-line stars (excluding Walborn's Of types), objects showing evidence for rapid rotation or line blending (nn types), and B-type stars of uncertain temperature or luminosity class (colon or double colon types), were excluded from the analysis by reason of having possibly unreliable intrinsic colors. Most other objects were included, however, in order to keep the sample sizes as large as possible. Using these criteria, we were readily able to identify six galactic regions that could be used to study the reddening law for OB stars.

Our adopted intrinsic colors for all program stars are based upon tables of $(B-V)_{0}$ and $(U-B)_{0}$ for early-type stars produced by blending FitzGerald's(1970) colors with the less detailed grid of Johnson (1966). These colors appear to give quite consistent results for O-, B-, and A-type stars (see Turner 1985). Johnson's values were derived from cluster stars of known reddening under the assumption that there are intrinsic variations in the interstellar reddening law, while FitzGerald adopted Johnson's $(B-V)_{0}$ values while solving for $(U-B)_{0}$ using two-color reddening lines of specified slope and curvature. Despite these different philosophical approaches to the problem, there are insignificant differences in the intrinsic colors tabulated by Johnson and FitzGerald for most early-type stars. We stress this point in order to demonstrate that our use of these intrinsic colors should not bias the analysis toward reddening lines of any particular slope or curvature. Various alternative sources of intrinsic colors for early-type stars can be found in the literature, but it is not always clear how they were derived. Since most list nearly identical values to those used here, the present results should depend very little upon the exact choice of intrinsic colors.

Table I identifies the six regions analyzed in this paper. These regions correspond to fields of open clusters and associations in which significant differential reddening is present. The references for the photometry and spectral types are quite extensive, and include some unpublished observations; they are not listed here for reasons of brevity. Each region is identified by its galactic coordinates, and Table I lists the sample sizes and the resulting values of $X$ and $Y$ (with their associated uncertainties) obtained in the reddening line analysis. Reddening data for individual stars in each region are plotted in Figs. 1-6, along with the reddening line solutions.

In fitting regression lines to the color excess data for each region, we assumed that the uncertainties in the values of $\mathrm{E}_{U-B}$ were roughly twice those in the values of $\mathrm{E}_{B-V}$, and also that the best-fitting line passed through the origin. This last assumption was felt to be justified by the careful selection of input $U B V$ data (which should be free of systematic errors) and the use of well-defined intrinsic colors. Without it the curvature of each best-fitting line tends to become slightly larger, and results in spurious zero-point corrections for the colors of individual stars. Alternative best-fitting reddening lines that do not pass through the origin of each plot are not presented here since they are considered to be unrepresentative of the actual relationships.

\section{RESULTS}

The reddening line parameters tabulated in Table I are plotted as a function of galactic longitude in Fig. 7. Despite some scatter in the reddening plots of Figs. 1-6, the individual parameters $X$ and $Y$ for each region are reasonably well established to within \pm 0.01 or \pm 0.02 . The weighted mean values for all six regions are $\langle\bar{X}\rangle=0.724 \pm 0.005$ and $\langle Y\rangle=0.021 \pm 0.006$. It is important to note that all of the individual $Y$ estimates agree to within their uncertainties with the mean $\langle Y\rangle$, but only one of the six $X$ estimates lies close to the mean $\langle X\rangle$. The straightforward interpretation of this result is that the curvature in reddening lines for earlytype stars is both small and uniquely defined, but the slope is variable from one region of the Milky Way to another. This

TABLE I. Reddening line parameters.

\begin{tabular}{lcccc}
\hline \hline Region & $l\left(^{\circ}\right)$ & $b\left(^{\circ}\right)$ & Stars & $X \pm$ s.e. \\
\hline NGC 6611 and Sgr OB1 & 17.0 & +0.8 & 69 & $0.721 \pm 0.015$ \\
Vul OB1 and Vul OB2 & 64.0 & +0.2 & 30 & $0.740 \pm 0.011$ \\
Cyg OB2 & 80.1 & +0.9 & 71 & $0.797 \pm 0.018$ \\
NGC 2439 and Anon Pup OB & 247.2 & -4.0 & 25 & $0.754 \pm 0.014$ \\
Sco OB2 & 350.0 & +20.0 & 46 & $0.622 \pm 0.011$ \\
Trumpler 27 & 355.1 & -0.7 & 48 & $0.793 \pm 0.014$ \\
\hline \hline
\end{tabular}




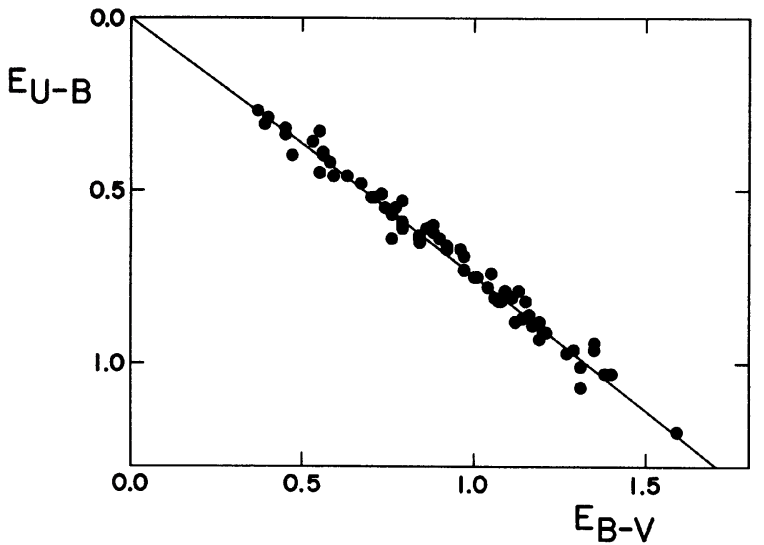

FIG. 1. Color excess data and best-fitting relation for early-type stars in the field of NGC 6611 and Sgr OBI.

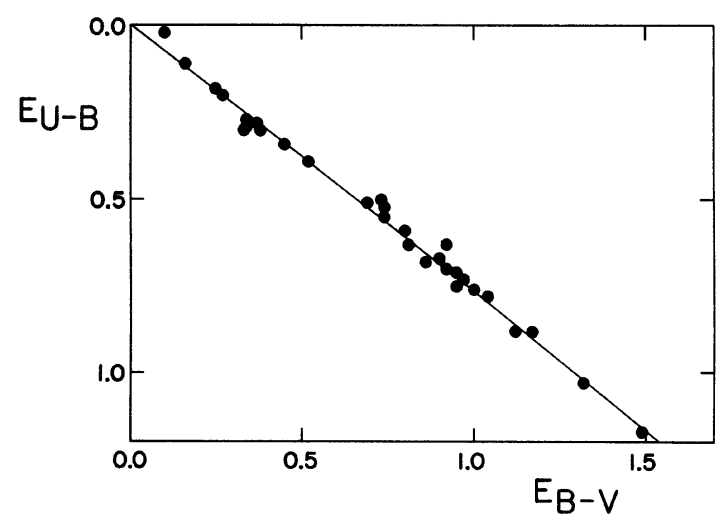

FIG. 2. Same as Fig. 1 for the field of $\mathrm{Vul} \mathrm{OBl}$ and $\mathrm{Vul} \mathrm{OB2.}$

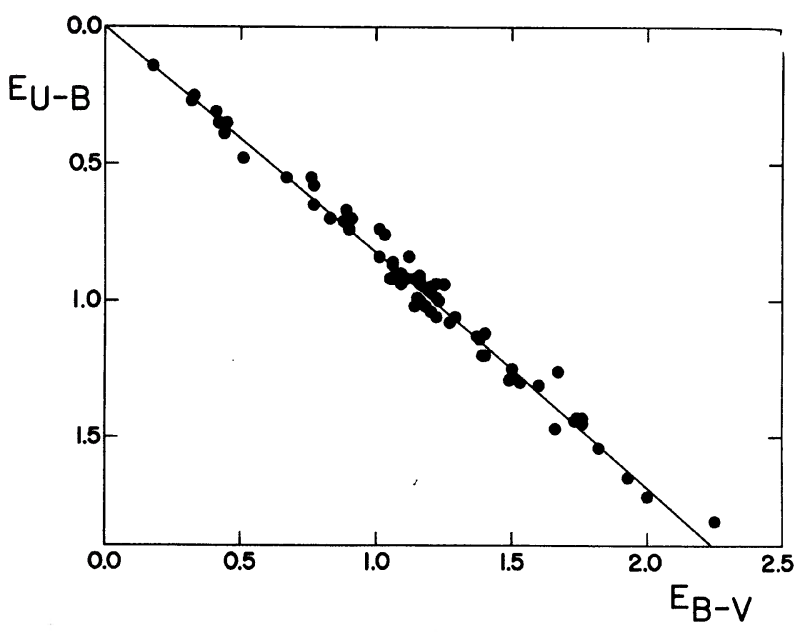

Fig. 3. Same as Fig. 1 for the field of Cyg OB2.

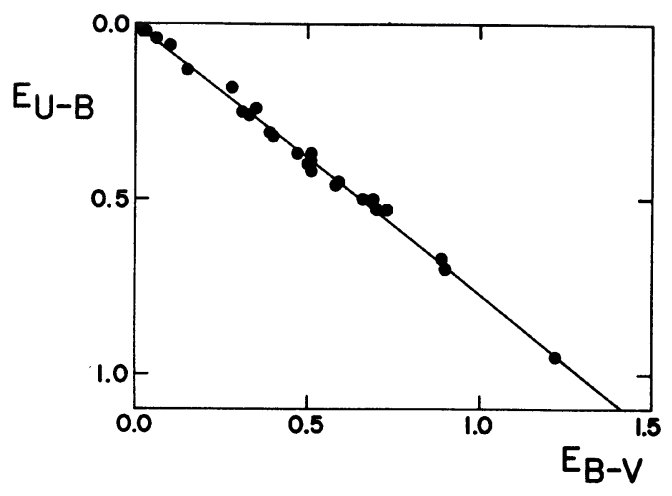

Fig. 4. Same as Fig. 1 for the field of NGC 2439 in Puppis.

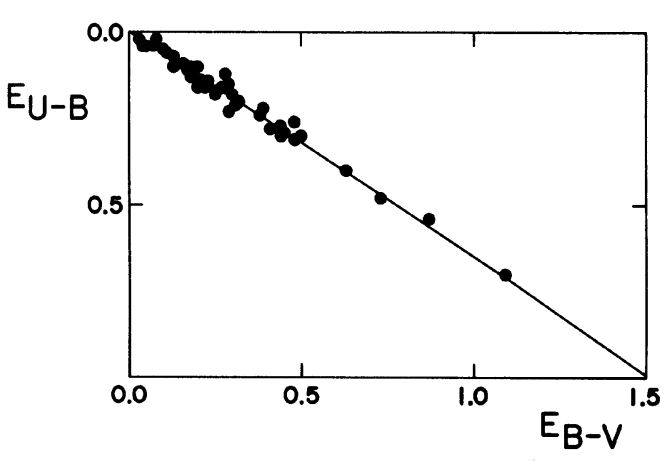

Fig. 5. Same as Fig. 1 for the field of Sco OB2.

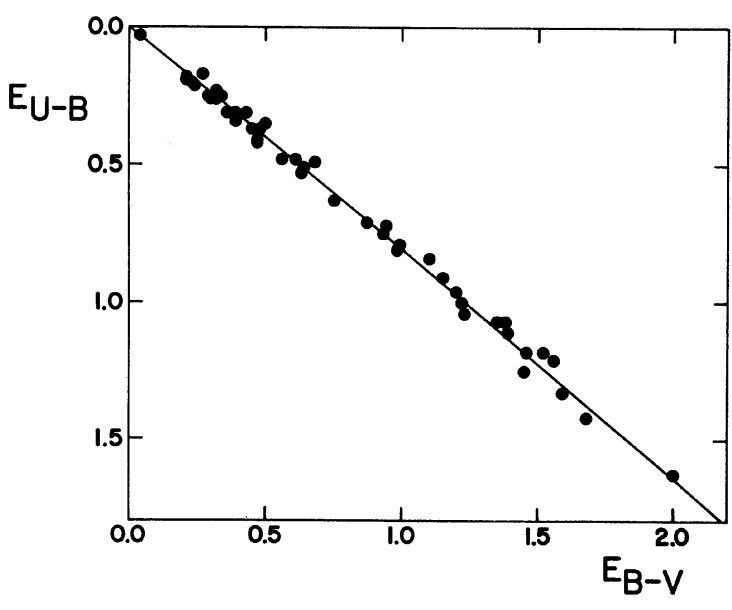

Fig. 6. Same as Fig. 1 for the field of Trumpler 27. 


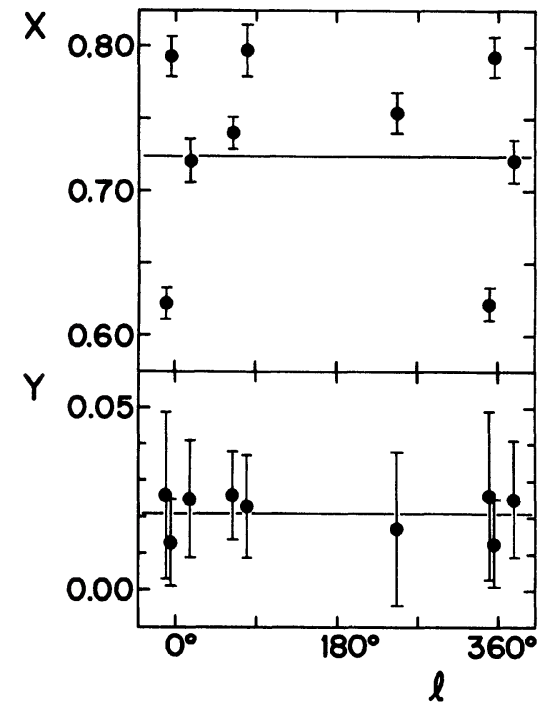

Fig. 7. Derived reddening line slope $(X)$ and curvature $(Y)$ parameters (and their uncertainties) plotted as a function of galactic longitude.

last conclusion is valid even when the rather small reddening slope for Sco OB2 is excluded. In this case $\langle X\rangle$ (without Sco OB2 $)=0.757 \pm 0.006$, with again only one of the five remaining $X$ estimates lying close to the mean. The average reddening line slope of $X \simeq 0.72$ is identical to the value originally obtained by Hiltner and Johnson (1956), but the curvature term of $Y \simeq 0.02$ is less than half as large as the values most frequently quoted for it in the literature.

It might be argued that the methodology employed in obtaining the reddening line parameters of Table I and Fig. 7 must somehow be erroneous, since the results seem to be at variance both with theoretical predictions and with the conclusions reached in those observational studies using large statistical samples. It is our feeling that the results of Serkowski (1963) and FitzGerald (1970) have probably been strongly influenced by undesirable systematic effects in the color excess data, whereas the theoretical predictions for reddening in the $U B V$ system are more likely to be qualitatively, rather than quantitatively, correct.

The primary source of systematic error in reddening line analyses for early-type stars (other than systematic errors in the photometry) most likely lies in the spectral classifications, which are an inhomogeneous sample that can be seriously affected by the use of nonstandard techniques or inadequate standard stars (Garrison 1985). This is most important for dwarf stars of spectral types B0.5-A0, where the spectroscopic characteristics at MK dispersion that distinguish stars of one temperature subtype from those of successively later temperature subtypes are weak lines and blends, the visibility of which is frequently diminished by various observational and intrinsic factors (e.g., instrumental focus, seeing, dispersion, spectral widening, exposure time, stellar rotation, chemical peculiarities). It is not uncommon to find stars of spectral types B0.5 V to A0 V that have been misclassified toward systematically later spectral types by researchers whose spectroscopic observations do not satisfy all of the standard criteria for MK classification.

This effect is apparent in Fig. 8, which plots the color

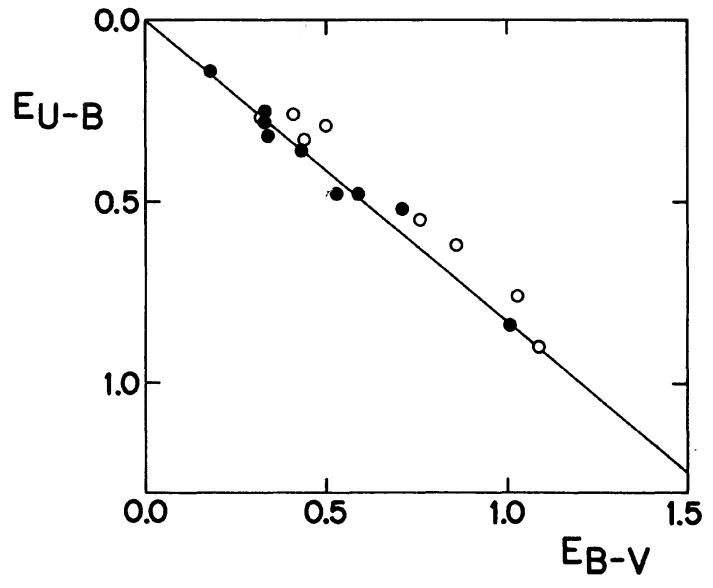

FIG. 8. Color excess data and best-fitting straight line for B1 V to B3 V stars in the field of Cyg OB2. Filled circles represent stars with good quality MK spectral classifications; open circles represent stars with MK types of uncertain quality.

excesses for stars in the Cyg OB2 region that have been classified as B1 V, B2 V, or B3 V. Stars classified by researchers using classical MK techniques (including five by Morgan himself) are denoted by filled circles, while stars with MK types from papers by researchers less experienced in spectral classification are denoted by open circles. The straight line of slope $X=0.826( \pm 0.014)$ is an alternative fit to the Cyg $\mathrm{OB} 2$ data (assuming $Y=0.00$ ). Clearly, the B1 V to B3 V stars fit the reddening relation established for stars in the Cyg OB2 region of earlier type, provided that one considers only the subset of stars classified in rigorous fashion ( $X=0.821 \pm 0.068$ for these stars). This conclusion is seemingly not true if one considers the larger sample of stars that have MK spectral classifications in the B1 V to B3 V range. Indeed, most of the extra data points in Fig. 8 lie above the reddening relation for this field, implying a smaller reddening slope for these stars $(X=0.776 \pm 0.050$ for the entire sample). The discrepancy is actually quite easily resolved by assuming that the lower-quality MK types are systematically misclassified about one spectral subtype cooler on average than the true spectral subtypes for the stars, a situation arising in the literature more frequently than one would wish. Under this assumption, all of the sample of B1 V to B3 V stars would lie close to the standard Cyg OB2 reddening line, with no obvious change in slope $X$ over the interval O3-B3.

In the alternative scenario, in which one does not discount stars on the basis of the source of the MK spectral classification, the interpretation forced on the researcher is that the reddening line for $\mathrm{B} 1 \mathrm{~V}$ to $\mathrm{B} 3 \mathrm{~V}$ stars is of different slope from that for stars of earlier type. For the data in Fig. 8, a reddening slope $X=X_{\mathrm{OB}}-0.05$ provides a reasonably good fit to the data (the slope of the reddening line for B1$\mathrm{B} 3$ stars would be about 0.05 less than its value for O3-B0.5 stars). This reasoning was used previously by Lindholm (1957), Serkowski (1963), and FitzGerald (1970) to derive a spectral type dependence of $X$ for B stars. However, the effect is not nearly as pronounced in theoretical simulations (e.g., Buser 1978) as in these observational studies, and may be negligible. The data of Fig. 8 argue quite effectively for the need of a new investigation of this problem based upon goodquality MK types only. 
Figure 9 depicts reddening slopes for individual groups of stars of specific spectral type (B1, B2,.., A7) in the Sco OB2 region, where the classifications represent a homogeneous sample from Garrison (1967), obtained using rigorous MK techniques. Simple straight-line fits were made to the two-color $U B V$ data using a combination of multivariate and nonparametric techniques, and the resulting solutions for $X$ (and their associated uncertainties) have been plotted in Fig. 9 as a function of the intrinsic $B-V$ color for the spectral type common to each group. A value of $X=0.631$ $( \pm 0.014)$ applies to Sco OB2 stars under the assumption that $Y=0.00$, and a reddening slope of this value is plotted in Fig. 9 for comparison with the individual straight-line fits. It can be seen that there are no obvious spectral-type-dependent trends in the reddening slopes relative to this value over the interval B1-A7, certainly none of the type found by Lindholm, Serkowski, and FitzGerald. A less marked dependence of $X$ on intrinsic color of the type obtained by Buser (1978) in his theoretical study cannot be discounted, although Buser's values require a systematic correction in order to match the smaller overall value of $X$ for the Sco OB2 region. A Buser-type spectral class dependence of $X$ and a constant value over the interval B1-A7 both fit the data of Fig. 9 with equal success ( 10 of 12 points matched), and it is not possible to determine which solution is more appropriate.

It would be particularly useful to have similar plots for other regions of the Milky Way, but the necessary data simply do not exist. To the extent that the results for Sco OB2 are representative of other regions, one can reasonably conclude that any spectral type dependence of $X$ for early-type stars is not marked. The actual variation in $X$ over the interval B1-A7 in Fig. 9 appears to be less than $10 \%$, and may be negligible. A single value for the reddening slope $X$ might therefore be entirely appropriate for O-, B-, and A-type stars in localized regions of the Milky Way, without invoking the need for a complicated spectral-type dependence that may not be justified. Very similar conclusions were reached by

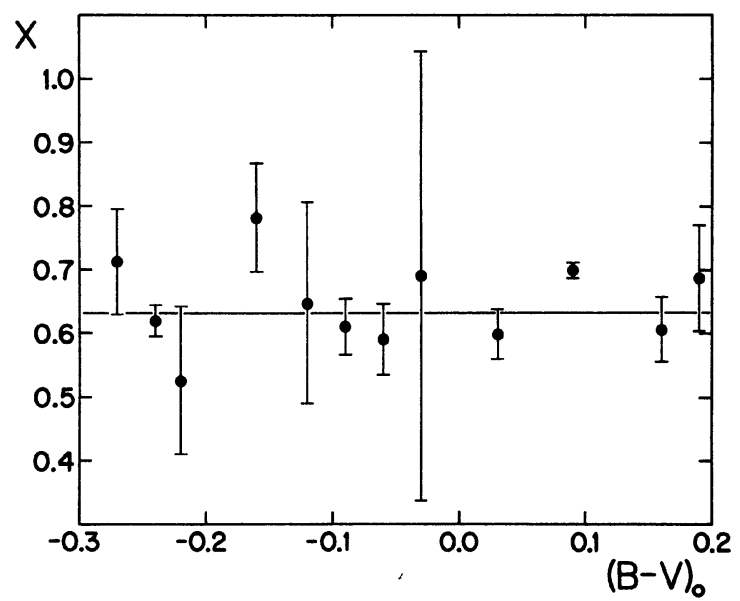

FIG. 9. Derived group reddening slopes (and their uncertainties) for dwarf stars in the Sco OB2 region of types B1, B2, .., A7, plotted as a function of the intrinsic color of the spectral subtype representing each group. The horizontal line is the best-fitting, straight-line, reddening slope for the Sco OB2 field.
Mawridis (1958) over 30 years ago, and we reiterate them here.

The curvature term $Y$ found here is quite small compared to theoretical predictions, and appears to be almost negligible. The same systematic errors in spectral classification that can lead to apparent spectral type dependences for $X$ may be responsible for a systematic scatter above the reddening lines of Figs. 1-6. If so, $Y$ may actually be smaller than the value $(0.02)$ obtained in this study. We examined our stellar data samples for the six selected galactic regions studied, but could not determine exactly how important a small spectral type dependence for $X$ and the accidental inclusion of misclassified stars in the samples would be to the derived results. It is our impression that these effects are negligible, particularly in regions such as Cyg $\mathrm{O} 82$ that are dominated by $\mathrm{O}$ type stars. However, a future multivariate analysis that includes additional regions and examines the data for more restricted spectral ranges may provide a more convincing and reliable result in this regard. It seems unlikely that such factors affect the present analysis to such an extent as to invalidate the main conclusions. A curvature term of order $Y \simeq 0.02$ and a slope $X$ that varies from one galactic region to another over a range of at least $0.62-0.80$ appear to be quite representative observational parameters for the galactic reddening curve. Over small ranges of reddening the differences between reddening lines of curvature $Y=0.02$ and $Y=0.00$ are negligible, and the dereddening of $U B V$ data for O-, B-, and A-type stars in the same field can be handled quite effectively using straight lines of slope $X$ fixed by color excess data $\left(\mathrm{E}_{U-B}, \mathrm{E}_{B-V}\right)$ for stars in the region (cf. Turner 1981a,b).

\section{DISCUSSION}

The conclusions reached in this study can be summarized as follows: (i) the curvature of interstellar reddening lines for early-type stars is quite small ( $Y \simeq 0.02$ ), and is negligible over small color excess intervals; (ii) the slope of interstellar $U B V$ reddening lines varies quite dramatically from one region of the Milky Way to another. Use of published "mean" interstellar reddening lines is not recommended, since they will most likely be invalid for the region of sky being studied. This last conclusion has been reached many times before in the literature, but has apparently not been given the prominence it deserves.

Although $X$ is confirmed to be variable in this study, there is no obvious galactic longitude dependence of this parameter discernible in Fig. 7. This can presumably be explained by the fact that only three of the six regions studied (NGC 6611, Cyg OB2, and Sco OB2) are reddened predominantly by nearby dust associated with Gould's Belt; the remaining three regions are reddened mostly by more distant dust complexes. According to Whittet (1979), nearby dust associated with Gould's Belt produces a distinct galactic longitude dependence in the wavelength of maximum polarization, $\lambda_{\max }$, that is not seen in more distant dust complexes. Variations in $\lambda_{\max }$ around the Milky Way can be attributed to variations in the size distribution of particles producing interstellar polarization, with the dust associated with Gould's Belt being characterized by enhanced particle sizes. Likewise, variations in reddening slope $X$ can be produced by similar variations in the size distribution of reddening particles along the various galactic lines of sight. A predominance of large particles (neutral absorbers), for example, should act to reduce the value of $X$, whereas small particles (highly selective absorbers) should act to increase the value of $X$. 


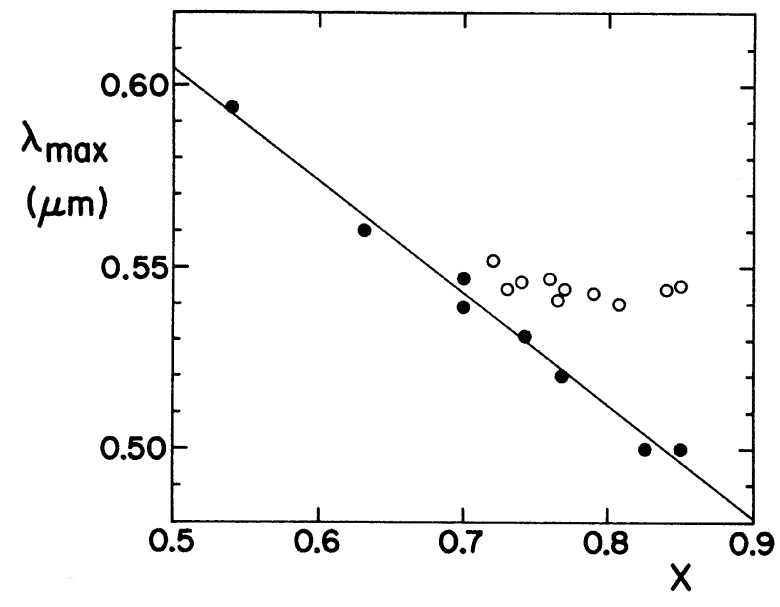

FIG. 10. Plot of the wavelength of maximum polarization versus reddening slope for regions of the Milky Way associated with open cluster fields. Filled circles represent regions reddened by nearby $(d<500 \mathrm{pc})$ dust (with best-fitting relation); open circles represent regions reddened by distant dust complexes.

A possible link between particle size distribution and reddening slope is evident in Fig. 10, which plots $\lambda_{\max }$ for several galactic regions versus derived reddening slope $X$ for that region. The values of $X$ have been taken from Table II, which presents alternative straight-line solutions for the six regions of Table I, and from various published and unpublished results for open cluster fields. The values of $\lambda_{\max }$ have been taken from Whittet (1979), using interpolation within his Table I for the distance interval appropriate for the distance of the reddening clouds in each field. The reddening clouds for several regions (NGC 6611, Cyg OB2, Sco OB2, BB Sgr, Roslund 3, SU Cas, NGC 1647, Mon R2, and NGC 3532) are close enough that the reddening slope correlates directly
TABLE II. Straight-line reddening slopes.

\begin{tabular}{lcccc}
\hline \hline Region & $l\left(^{\circ}\right)$ & $b\left(^{\circ}\right)$ & Stars & $X \pm$ s.e. \\
\hline NGC 6611 and Sgr OB1 & 17.0 & +0.8 & 69 & $0.742 \pm 0.014$ \\
Vul OB1 and Vul OB2 & 64.0 & +0.2 & 30 & $0.759 \pm 0.016$ \\
Cyg OB2 & 80.1 & +0.9 & 71 & $0.826 \pm 0.014$ \\
NGC 2439 and Anon Pup OB & 247.2 & -4.0 & 25 & $0.765 \pm 0.013$ \\
Sco OB2 & 350.0 & +20.0 & 46 & $0.631 \pm 0.014$ \\
Trumpler 27 & 355.1 & -0.7 & 48 & $0.807 \pm 0.010$ \\
\hline \hline
\end{tabular}

with the value of $\lambda_{\max }$ assumed to apply in the field. The best-fitting line drawn in Fig. 10 for these fields is $\lambda_{\max }(\mu \mathrm{m})=0.76-0.31 X$. The remaining fields are distant enough that existing polarization data do not give $\lambda_{\max }$ values that are well correlated with $X$. This is reasonable given the lack of polarization observations for stars in these specific regions and the fact that the properties of polarization and reddening along specific lines of sight are cumulative effects that for polarization measures are strongly influenced by the properties of relatively nearby dust particles. Typical "distant" groups exhibit reddening slopes of $X=0.72$ to 0.85 , which should correlate with $\lambda_{\max }$ values in the range 0.50 0.54, indicative of "standard" particle sizes. According to the polarization observations (Whittet 1979), the dust associated with Gould's Belt has enhanced grain sizes relative to those typical of the dust in more distant regions of the Galaxy. Our present results for the variation of $X$ throughout the Milky Way are completely consistent with this conclusion.

This study was supported by research funding provided through the Natural Sciences and Engineering Research Council of Canada and through the Research Committee of the Saint Mary's University Senate. It is a pleasure to acknowledge Jim Moorhead and Bob Garrison for many illuminating (and formative) comments they provided in the very early stages of this study.

\section{REFERENCES}

Blanco, V. M. (1956). Astrophys. J. 123, 64.

Blanco, V. M. (1957). Astrophys. J. 125, 209.

Buser, R. (1978). Astron. Astrophys. 62, 411.

Crawford, D. L., and Mandwewala, N. (1976). Publ. Astron. Soc. Pac. 88, 917.

FitzGerald, M. P. (1970). Astron. Astrophys. 4, 234.

Garrison, R. F. (1967). Astrophys. J. 147, 1003.

Garrison, R. F. (1985). In Calibration of Fundamental Stellar Quantities, IAU Symposium No. 111, edited by D. S. Hayes, L. E. Pasinetti, and A. G. Davis Philip (Reidel, Dordrecht), p. 17.

Gutiérrez-Moreno, A., and Moreno, H. (1975). Publ. Astron. Soc. Pac. 87 425.

Hiltner, W. A., and Johnson, H. L. (1956). Astrophys. J. 124, 367.

Johnson, H. L. (1963). In Basic Astronomical Data, edited by K. Aa. Strand (University of Chicago, Chicago), Chap. 11.
Johnson, H. L. (1966). Annu. Rev. Astron. Astrophys. 4, 193.

Johnson, H. L., and Morgan, W. W. (1953). Astrophys. J. 117, 313.

Lindholm, E. H. (1957). Astrophys. J. 126, 588.

Mawridis, L. (1958). Z. Astrophys. 45, 98.

Popper, D. M. (1982). Publ. Astron. Soc. Pac. 94, 204.

Serkowski, K. (1963). Astrophys. J. 138, 1035.

Straizys, V., Sudzius, J., and Kuriliene, G. (1976). Astron. Astrophys. 50, 413.

Turner, D. G. (1981a). Astron. J. 86, 222.

Turner, D. G. (1981b). Astron. J. 86, 231.

Turner, D. G. (1985). Astrophys. J. 292, 148.

Wampler, E. J. (1961). Astrophys. J. 134, 861.

Wampler, E. J. (1962). Astrophys. J. 136, 100.

Wampler, E. J. (1964). Astrophys. J. 140, 1615.

Whittet, D. C. B. (1979). Astron. Astrophys. 72, 370. 